\title{
HELIUM REFRIGERATOR LIQUID NITROGEN PRE-COOLER COMPONENT PARAMETER SENSITIVITY ANALYSIS
}

\author{
P. Knudsen, V. Ganni \\ Thomas Jefferson National Accelerator Facility \\ Newport News, Virginia, 23606, USA
}

\begin{abstract}
It is not uncommon for helium refrigerators using liquid nitrogen (LN) pre-cooling to exhibit nitrogen usage significantly higher than estimated by the design. Often this is attributed to an underperforming 300 to 80 -K helium-nitrogen heat exchanger(s) rather than the 300 to $80-\mathrm{K}$ helium-helium heat exchanger(s), even though the return helium stream(s) exiting the cold box at the warm (ambient) end are significantly colder than the design goals. Furthermore, typically there is an specious emphasis on the size of the 300 to $80-\mathrm{K}$ helium-nitrogen heat exchanger(s) to ensure the exiting nitrogen is as close to ambient temperature as possible, presumably to reduce the LN usage. However, in fact, it is the performance resulting from the size and the flow distribution of the 300 to $80-\mathrm{K}$ heliumhelium heat exchangers which has the prevalent influence on the nitrogen consumption. This paper examines the sensitivity of the key parameters in the design and operation of a refrigerator $\mathrm{LN}$ pre-cooler section affecting the LN consumption.
\end{abstract}

KEYWORDS: helium, refrigerator, liquid nitrogen, pre-cooling

\section{INTRODUCTION}

The use of liquid nitrogen (LN) pre-cooling in helium refrigeration systems is very common. It is quite efficacious and beneficial as it reduces the investment cost of the system for a given capacity, results in fewer expanders, smaller size cold box, smaller compressor system and provides a stable thermal anchor point for the $80-\mathrm{K}$ cold box adsorber beds for contamination prevention to the colder parts of the system. Systems with LN pre-cooling are useful in handling the cool-down of large loads and during partial cold box maintenance where the load is kept at $80 \mathrm{~K}$. However, using additional turbines, rather than LN pre-cooling, eliminates the risk of nitrogen delivery problems (due to weather, summer power restrictions or nitrogen plant problems), usually results in lower operating 
costs, prevents the danger of nitrogen cross-leakage into helium and prevents additional oxygen deficiency hazards.

For systems using LN pre-cooling it is highly desirable to minimize the amount of nitrogen used. Therefore, it is of prime importance to identify key component parameters in the design of systems using LN pre-cooling and key process parameters for operating these systems that will accomplish this objective [1]. Often, there is an emphasis on the size of the 300 to $80-\mathrm{K}$ helium-nitrogen $\mathrm{HX}$ in design. Likewise, in operation, there is an emphasis on ensuring that the (absolute) temperature of the exiting gaseous nitrogen is close to ambient. However, it is very common to find operating systems whose nitrogen consumption is significantly higher than projected by the ('TS') design. Coincidentally, but not unrelated, in these systems the return helium stream(s) exiting the warm-end of the 300 to $80-\mathrm{K}$ helium-helium HX is substantially colder than intended in the ('TS') design.

There are basically two types of LN pre-cooling utilized as shown in FIGURE 1. Type 1, or direct LN injection, does not employ phase separation. This type is commonly used in smaller systems. Type 2 (and its various sub-types) employ phase-separation and are more commonly used on larger systems. Further sub-classifications of Type 2 systems are as follows:

TYPE 1

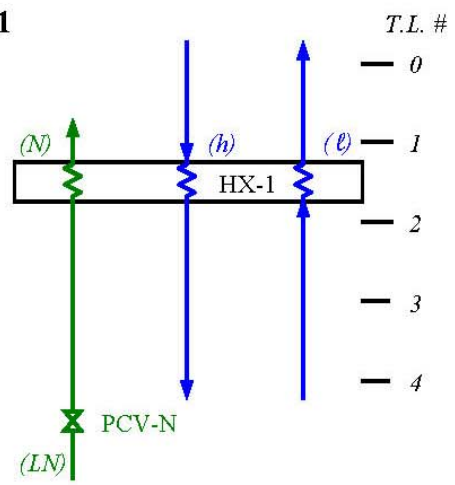

TYPE 2-F(T)

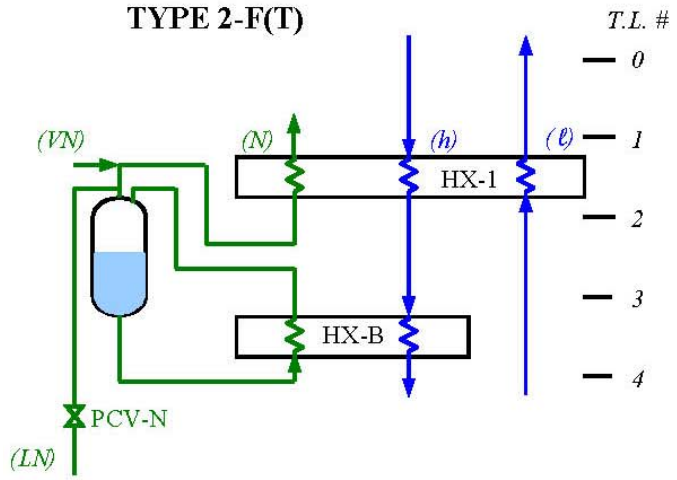

TYPE 2-B(O)

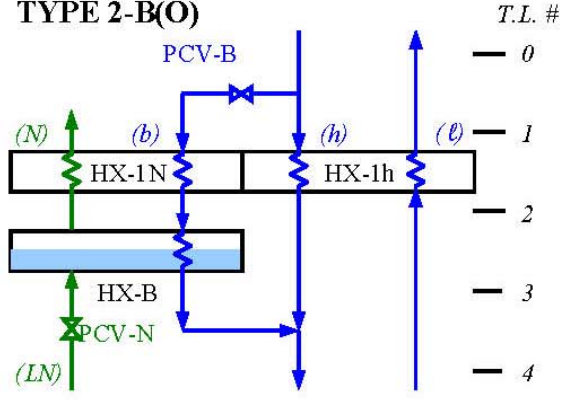

TYPE 2-BF(T)

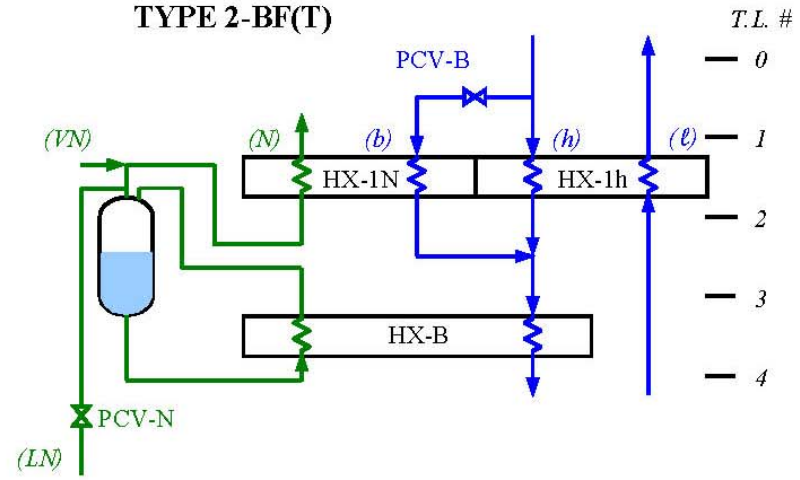

TYPE 2-BF(O)

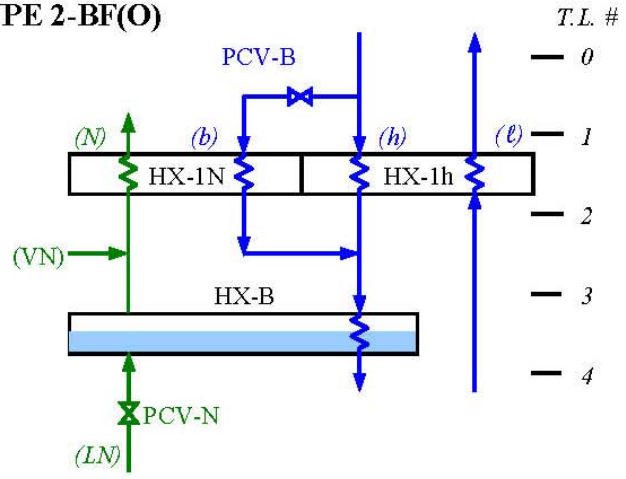

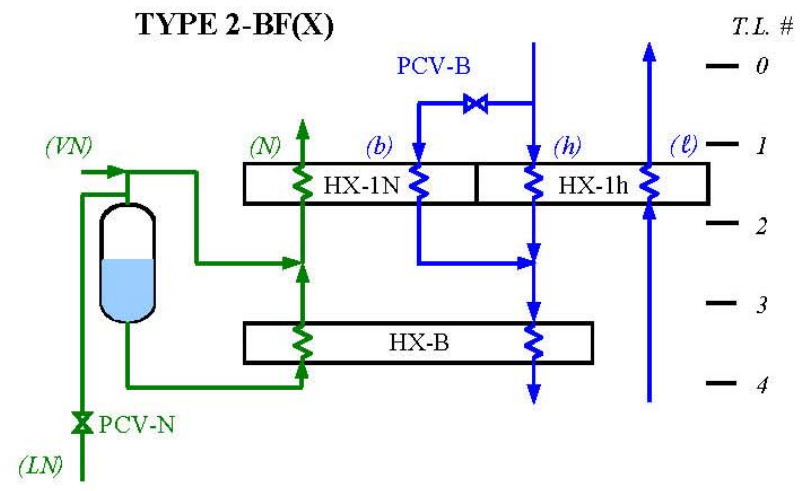

FIGURE 1. Process Flow Diagrams - Open Type Boiler and Thermo-Siphon (Respectively) 
a. Distinct separation of the high pressure helium flow being cooled into a main stream and a 'bypass' stream (i.e., 'B') which is in counter-flow to the nitrogen

b. Total high pressure helium flow cooled by boiler (i.e., ' $F$ '), or only part of the total (i.e., the 'bypass' stream) flow

c. 'Open' boiler (bath) vs. thermo-siphon \{i.e., '(O)’ or '(T)’\}

Note that Type 2-BF(X) is really feigning as a Type 2 pre-cooler since there is effectively no phase-separation with the two-phase exiting the boiler section (HX-B) entering the (presumed) sensible section $(\mathrm{HX}-1 \mathrm{~N})$. Proper phase-separation could be easily and inexpensively accomplished by sending the two-phase exiting the boiler section back to the liquid vessel and adding a vapor line from the liquid vessel to the sensible HX section $\{$ as in Type 2-F(T) and 2-BF(T)\}. In such cases, stable control is inherently difficult and has similar problems to a Type 1 arrangement (as will be discussed). Although Type 2-B(O) employs phase-separation, the total high pressure helium flow is not cooled by the boiler section. As such, it exhibits a cooling curve that is similar to a Type 1 pre-cooler (as will be discussed), with a very large helium-nitrogen stream temperature difference (on the order of $100 \mathrm{~K}$ ) between the latent and sensible HX sections. This is a consequence of nitrogen's latent heat being close to its sensible enthalpy. It is important that the boiler (latent HX) section for this type of pre-cooler be properly designed for film boiling.

There is typically one control element to essentially affect LN usage. For Type 1 precoolers this is 'PCV-N' (i.e., the LN supply valve). For Type 2 pre-coolers with a bypass stream this is 'PCV-B' (i.e., the high pressure helium bypass stream valve). Type 2 precoolers without a bypass stream do not have a means to affect the LN usage, as 'PCV-N' is used to maintain liquid level in the phase-separator.

\section{LN PRE-COOLER DESIGN}

Normally for the LN pre-cooler design, the warm-end and cold-end stream temperature differences are specified. This determines the design LN usage (i.e., supply flow rate). That is,

$$
\xi_{L N}=\frac{\left[\left(\Delta h_{h l, 0}-\Delta h_{h l, 4}\right)+\xi_{L} \cdot \Delta h_{l, 04}+\left(\sum q_{L K}\right) / \dot{m}_{h}\right]}{\left[\left(1-\xi_{V N}\right) \cdot \Delta h_{V N}+\Delta h_{L N}\right]}
$$

Where,

$$
\begin{gathered}
\dot{m}_{h}=\dot{m}_{h, 0}, \quad \dot{m}_{L}=\dot{m}_{h}-\dot{m}_{l}, \quad \dot{m}_{N}=\dot{m}_{L N}+\dot{m}_{V N} \\
\xi_{L N}=\dot{m}_{L N} / \dot{m}_{h}, \quad \xi_{V N}=\dot{m}_{V N} / \dot{m}_{L N}, \quad \xi_{L}=\dot{m}_{L} / \dot{m}_{h} \\
\Delta h_{h l, 0}=h_{h, 0}-h_{l, 0}, \Delta h_{h l, 4}=h_{h, 4}-h_{l, 4}, \quad \Delta h_{l, 04}=h_{l, 0}-h_{l, 4} \\
\Delta h_{V N}=h_{N, 0}-h_{V N}, \Delta h_{L N}=h_{V N}-h_{L N}=\left(1-x_{N}\right) \cdot \lambda
\end{gathered}
$$

Note that $\dot{m}_{V N}$ is saturated nitrogen vapor, which is sometimes added from an external source (i.e., $\xi_{V N}$ is specified). Also, $h_{L N}$ is the enthalpy of the supplied liquid nitrogen that is not necessarily at the same saturation pressure as in the boiler or phase-separator; so that, when it is throttled (at constant enthalpy) across PCV-N a vapor mass fraction of $x_{N}$ is generated. Equation (1) can be re-written as, 


$$
\xi_{L N}=\frac{\left[\left(C_{p, h l, 0} \cdot \Delta T_{h l, 0}-C_{p, h l, 4} \cdot \Delta T_{h l, 4}\right)+\xi_{L} \cdot C_{p, l, 04} \cdot \Delta T_{l, 04}+\left(\sum q_{L K}\right) / \dot{m}_{h}\right]}{\left[\left(1-\xi_{V N}\right) \cdot C_{p, V N} \cdot \Delta T_{V N}+\Delta h_{L N}\right]}
$$

Where,

$$
\begin{gathered}
T_{N, 2}=T_{N, 3}=T_{N, 4}=T_{V N}=\text { saturation temperature at pressure, } p_{N, 2}=p_{N, 3} \\
\Delta T_{h l, 0}=T_{h, 0}-T_{l, 0}, \Delta T_{h l, 4}=T_{h, 4}-T_{l, 4}, \Delta T_{l, 04}=T_{l, 0}-T_{l, 4}, \Delta T_{V N}=T_{N, 1}-T_{N, 2} \\
C_{p, h l, 0}=\Delta h_{h l, 0} / \Delta T_{h l, 0}, \quad C_{p, h l, 4}=\Delta h_{h l, 4} / \Delta T_{h l, 4} \\
C_{p, l, 04}=\Delta h_{l, 04} / \Delta T_{l, 04}, \quad C_{p, V N}=\Delta h_{V N} / \Delta T_{V N}
\end{gathered}
$$

\section{KEY PARAMETER SENSITIVITY}

As mentioned previously, there is essentially one control element to affect LN usage for Type 1 pre-coolers and Type 2 pre-coolers with a bypass stream. Noting that the $80-\mathrm{K}$ level cold-end helium-helium stream temperature difference $\left(\Delta T_{h l, 4}\right)$ is set by the (colder) HX below and taking cue from the LN pre-cooler energy balance, we will posit that, for either a Type 1 or Type 2 pre-cooler, it is the warm-end helium-helium stream temperature differences $\left(\Delta T_{h l, 1}\right)$ and/or the warm-end helium-nitrogen stream temperature difference $\left(\Delta T_{b N, 1}\right.$ or $\left.\Delta T_{h N, 1}\right)$ that governs the LN usage $\left(\xi_{L N}\right)$. To examine this, a sensitivity analysis can be performed to examine the effect of the warm-end stream temperature differences on the LN usage.

It is useful to begin by putting equation (2) in dimensionless form, with,

$$
\begin{aligned}
\tau_{h l, 1}=\Delta T_{h l, 1} / T_{h, 1}, \quad \tau_{b N, 1} & =\Delta T_{b N, 1} / T_{h, 1}, \quad \tau_{h l, 4}=\Delta T_{h l, 4} / T_{l, 4}, \quad \tau_{h N, 4}=\Delta T_{h N, 4} / T_{l, 4} \\
\alpha_{h l, 0}=C_{p, h l, 0} / C_{p}{ }^{\circ}, & \alpha_{h l, 4}=C_{p, h l, 4} / C_{p}{ }^{\circ}, \alpha_{l, 04}=C_{p, l, 04} / C_{p}{ }^{\circ}, \alpha_{V N}=C_{p, V N} / C_{p}{ }^{\circ} \\
\Theta=T_{h, 1} / T_{l, 4}, \quad v_{N} & =\Delta h_{L N} /\left(C_{p}{ }^{\circ} \cdot T_{h, 1}\right), \quad \varepsilon_{k}=\Sigma q_{L K} /\left(\dot{m}_{h} \cdot C_{p}{ }^{\circ} \cdot T_{h, 1}\right) \\
\xi_{L N} & =\left(B_{0} \cdot \tau_{h l, 1}+B_{1}\right) /\left(B_{2}-B_{3} \cdot \tau_{b N, 1}\right)
\end{aligned}
$$

So, the sensitivities are,

$$
\left(\frac{\partial \xi_{L N}}{\partial \tau_{h l, 1}}\right)=\frac{B_{0}}{\left(B_{2}-B_{3} \cdot \tau_{b N, 1}\right)}, \quad\left(\frac{\partial \xi_{L N}}{\partial \tau_{b N, 1}}\right)=\frac{\xi_{L N} \cdot B_{3}}{\left(B_{2}-B_{3} \cdot \tau_{b N, 1}\right)}
$$

Where,

$$
\begin{gathered}
B_{0}=\alpha_{h l, 1}-\xi_{L} \cdot \alpha_{l, 14}, \quad B_{1}=\left[\xi_{L} \cdot \alpha_{l, 14} \cdot(\Theta-1)-\alpha_{h l, 4} \cdot \tau_{h l, 4}\right] / \Theta+\varepsilon_{k} \\
B_{2}=\left(1+\xi_{V N}\right) \cdot \alpha_{V N} \cdot\left[1-\left(1-\tau_{h N, 4}+\tau_{h l, 4}\right) / \Theta\right]+v_{N}, \quad B_{3}=\left(1+\xi_{V N}\right) \cdot \alpha_{V N} \cdot \tau_{b N, 1}
\end{gathered}
$$

The ratio of the these sensitivities is,

$$
\left(\frac{\partial \xi_{L N}}{\partial \tau_{h l, 1}}\right) /\left(\frac{\partial \xi_{L N}}{\partial \tau_{b N, 1}}\right)=\frac{B_{0}}{\xi_{L N} \cdot B_{3}}
$$

So, for $\xi_{L}<<1, B_{0} \approx\left(1-\xi_{L}\right) \approx 1$ 
With, $\alpha_{V N}=1.06 / 5.19 \cong 0.20, \Theta=300 / 80=3.75, \quad v_{N}=0.10$ to 0.12 , depending on the LN supply pressure, $B_{2} \approx\left(1+\xi_{V N}\right) \cdot \alpha_{V N} \cdot[1-1 / \Theta]+v_{N} \approx\left(1+\xi_{V N}\right) \cdot(0.20) \cdot(0.73)+0.11$

For $\xi_{V N}<<1, B_{2} \approx 0.26$, and, $B_{3} \approx\left(1+\xi_{V N}\right) \cdot(0.20) \approx 0.20$

Ranging from refrigeration ( $\xi_{L N} \approx 6 \%$ ) to liquefaction ( $\xi_{L N} \approx 33 \%$ ), the ratio of the sensitivities is, $\left(\frac{\partial \xi_{L N}}{\partial \tau_{h l, 1}}\right) /\left(\frac{\partial \xi_{L N}}{\partial \tau_{b N, 1}}\right) \approx \frac{5}{\xi_{L N}} \approx 15$ to 80 , for liquefaction to refrigeration, respectively.

That is, depending on amount of liquefaction, the LN consumption is affected roughly 15 to 80 times more by every degree Kelvin increase in the warm-end helium-helium stream temperature difference $\left(\Delta T_{h l, 1}\right)$ as compared to every degree Kelvin increase in the warm-end helium-nitrogen stream temperature difference $\left(\Delta T_{b N, 1}\right.$ or $\left.\Delta T_{h N, 1}\right)$.

FIGURE 2 shows the effect of the warm-end helium-helium HX stream temperature difference $\left(\Delta T_{h l, 1}\right)$ on the LN usage for two extreme warm-end helium-nitrogen HX stream temperature differences $\left(\Delta T_{b N, 1}\right.$ or $\Delta T_{h N, 1}$ of $5 \mathrm{~K}$ and $\left.40 \mathrm{~K}\right)$. FIGURE 3 shows the effect of the same on the size of the helium-helium HX (i.e., HX-1h) and the helium-nitrogen HX (i.e., HX-1N). Although these are for particular cases, they demonstrate the resulting effect of the sensitivities discussed.

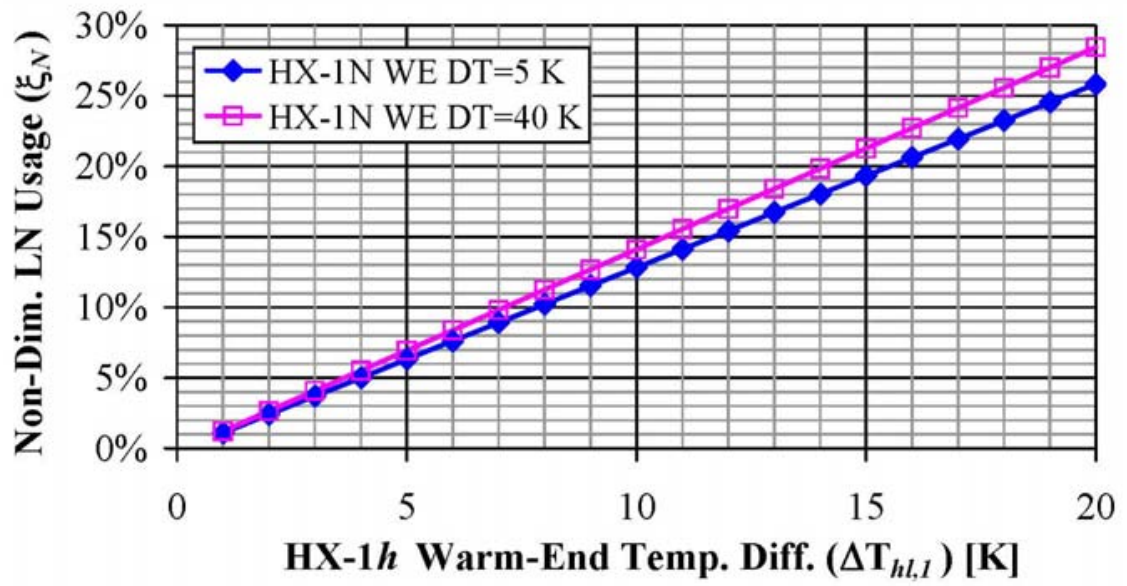

FIGURE 2. LN Usage vs. HX-1h Warm-End Temperature Difference

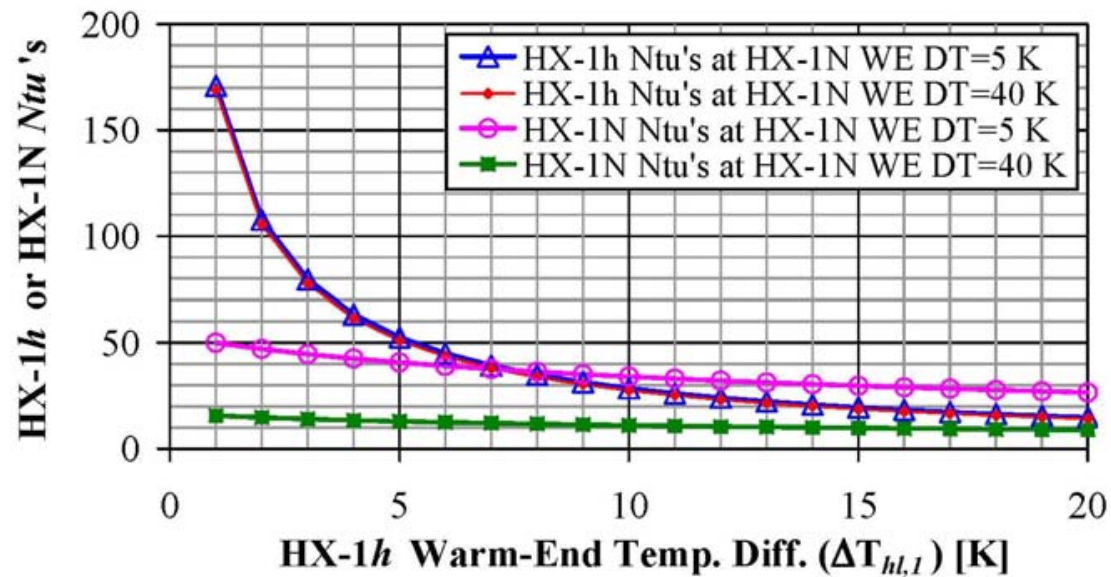

FIGURE 3. HX-1h and HX-1N Size vs. HX-1h Warm-End Temperature Difference 
TABLE 1. Nomenclature

\begin{tabular}{|lllll|}
\hline & Symbols: & \multicolumn{2}{l|}{ Subscripts: } \\
$C_{p}$ & Specific heat at constant pressure & {$[\mathrm{J} / \mathrm{g}-\mathrm{K}]$} & $b$ & High pressure helium bypass stream \\
$h$ & Enthalpy & {$[\mathrm{J} / \mathrm{g}]$} & $b N$ & Difference between ' $b$ ' \& ' $N$ ' streams \\
$\Delta h$ & Enthalpy difference & {$[\mathrm{J} / \mathrm{g}]$} & $h$ & High pressure helium stream \\
$\dot{m}$ & Mass flow rate & {$[\mathrm{g} / \mathrm{s}]$} & $h l$ & Difference between ' $h$ ' \& ' 'l' streams \\
$N t u$ & Number transfer units & {$[\mathrm{non}-\mathrm{dim}]$.} & $h N$ & Difference between ' $h$ ' \& ' $N$ ' streams \\
$q$ & Heat energy & {$[\mathrm{W}]$} & $l$ & Low pressure helium stream \\
$p$ & Pressure & {$[\mathrm{atm}]$} & $L$ & Liquefaction \\
$T$ & Temperature & {$[\mathrm{K}]$} & $L K$ & Heat leak \\
$\Delta T$ & Temperature difference & {$[\mathrm{K}]$} & $L N$ & Saturated liquid nitrogen supply \\
$x$ & Quality & {$[$ non-dim.] } & $m a x$ & Maximum \\
$\alpha$ & $C_{p}$ ratio with respect to $C_{p}{ }^{\circ}$ & {$[$ non-dim.] } & $N$ & Nitrogen stream \\
$\varepsilon$ & Non-dim. heat leak & {$[$ non-dim.] } & $V N$ & Saturated nitrogen vapor \\
$\lambda$ & Heat of vaporization & {$[\mathrm{J} / \mathrm{g}]$} & 1,2 etc. & Temperature level number \\
$\tau$ & Non-dim. stream temperature & {$[$ non-dim.] } & \multicolumn{3}{c}{} \\
$v$ & difference & Non-dim. effective latent heat & {$[$ non-dim.] } & $\underline{\text { Superscripts: }}$ \\
$\xi$ & Flow ratio & {$[$ non-dim.] } & \multicolumn{3}{c}{ Ideal, standard conditions } \\
\hline
\end{tabular}

\section{DIRECT LN INJECTION}

As previously mentioned, Type 1 pre-coolers are commonly used for smaller systems, presumably for simplicity and cost reasons. Since the latent cooling provided by nitrogen's heat of vaporization is approximately the same as the sensible cooling provided by the warmed vapor, and since only a partial portion of the high pressure helium flow is cooled by the latent portion of the nitrogen, the Type 1 pre-cooler manifests a HX cooling curve with a very high helium-nitrogen stream temperature difference $\left(\Delta T_{b N}\right)$ at the latentsensible boundary (internal to the HX). As can be demonstrated by a straightforward analysis, this stream temperature difference will not be lower than approximately $100 \mathrm{~K}$. As previously mentioned, although Type 2-B(O) utilizes phase separation, it shares this same characteristic since the total high pressure helium flow does not pass through the boiler section.

FIGURE 4 shows the cooling curve for a Type 1 (or Type 2-B) pre-cooler. This is somewhat idealized for a Type 1 pre-cooler, since the phase-boundary for flow boiling will not be as distinct as shown. As plainly seen, this is a very non-ideal cooling-curve. In principle a Type 1 and a Type-BF pre-cooler with the same warm-end and cold-end temperature differences have the same total exergetic losses. This is due to a trade-off between the higher losses in the former's helium-nitrogen section vs. higher losses in the latter's helium-helium section (because of higher mass flow). However, this very poor cooling curve will have quite a detrimental effect on the temperature and flow distribution in helium-helium passes in the Type 1 pre-cooler! Additionally, as FIGURE 4 indicates, the (approximate) location of the phase boundary, corresponding to the maximum heliumnitrogen stream temperature difference, moves as the nitrogen flow changes. FIGURE 5 shows this for two cases; a Type 1 (or Type 2-B) pre-cooler designed as a refrigerator, or one designed as a liquefier. As these figures indicate, a Type 1 pre-cooler is not inherently suited for stability or permissive of LN usage minimization. 


\section{TYPE 2 PRE-COOLERS WITH A HELIUM BYPASS PATH}

For a given design of a Type $2 \mathrm{LN}$ pre-cooler that has a separate helium bypass path and passes the total helium flow through the boiler section \{e.g., Type 2-BF(T) or Type 2$\mathrm{BF}(\mathrm{O})\}$, it is insightful to examine the effect of the helium bypass flow ratio $\left(\xi_{b}\right)$ on the LN usage $\left(\xi_{L N}\right)$. Although FIGURE 6 is for a specific case, it shows an important general behavior. There is a minimum LN usage at a unique bypass flow ratio. Also, the warmend stream temperature difference for $\operatorname{HX}-1 \mathrm{~N}\left(\Delta T_{b N, 1}\right)$ varies exponentially with the bypass flow ratio. In contrast, the warm-end stream temperature difference for $\mathrm{HX}-1 h\left(\Delta T_{h l, 1}\right)$ varies linearly and quite moderately. As such, stable control is more likely to the right of the minimum (i.e., labeled 'MIN' in FIGURE 6). So, to affect minimal LN usage for these pre-coolers, it appears that the most germane process variable that is able to satisfy this objective is the warm-end helium-helium $\mathrm{HX}$ temperature difference $\left(\Delta T_{h l, 1}\right)$.

\section{CONCLUSIONS}

As evident from the LN pre-cooler energy balance and sensitivity analysis, the stream temperature differences naturally arise from the mathematics as key variables. With this as a pre-cursor, it is important to emphasize the use of temperature differences for stable control, as opposed to the traditional practice of using absolute temperatures.

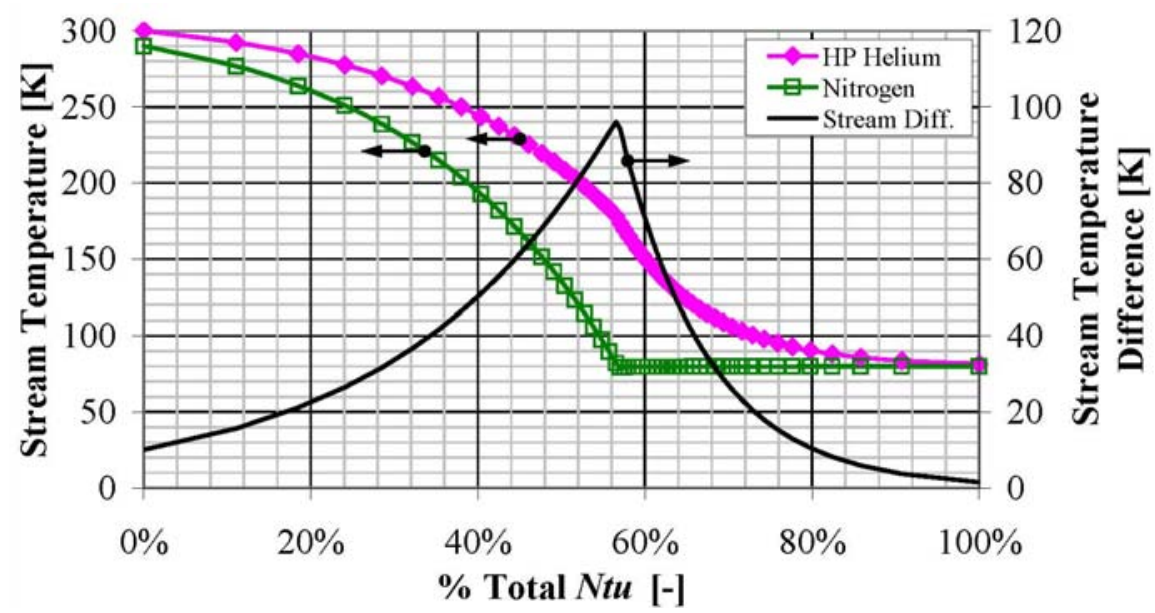

FIGURE 4. Type 1 and Type 2-B(O) Helium-Nitrogen Cooling Curve

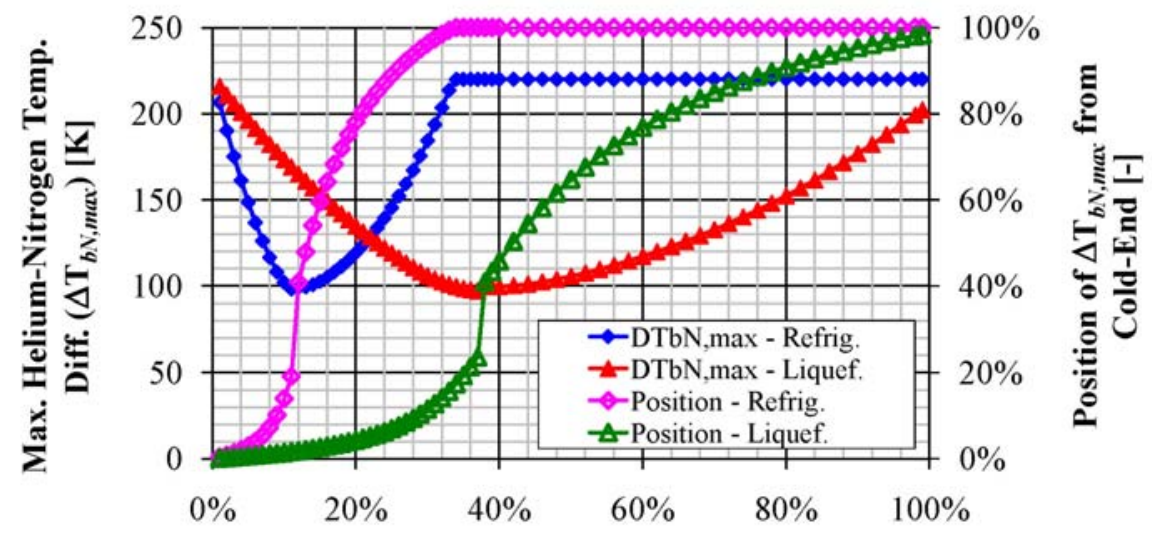

Non-Dim. LN Usage $\left(\xi_{N}\right)[-]$

FIGURE 5. Type 1 and Type 2-B(O) Maximum Helium-Nitrogen Stream $\Delta \mathrm{T}$ and Location 


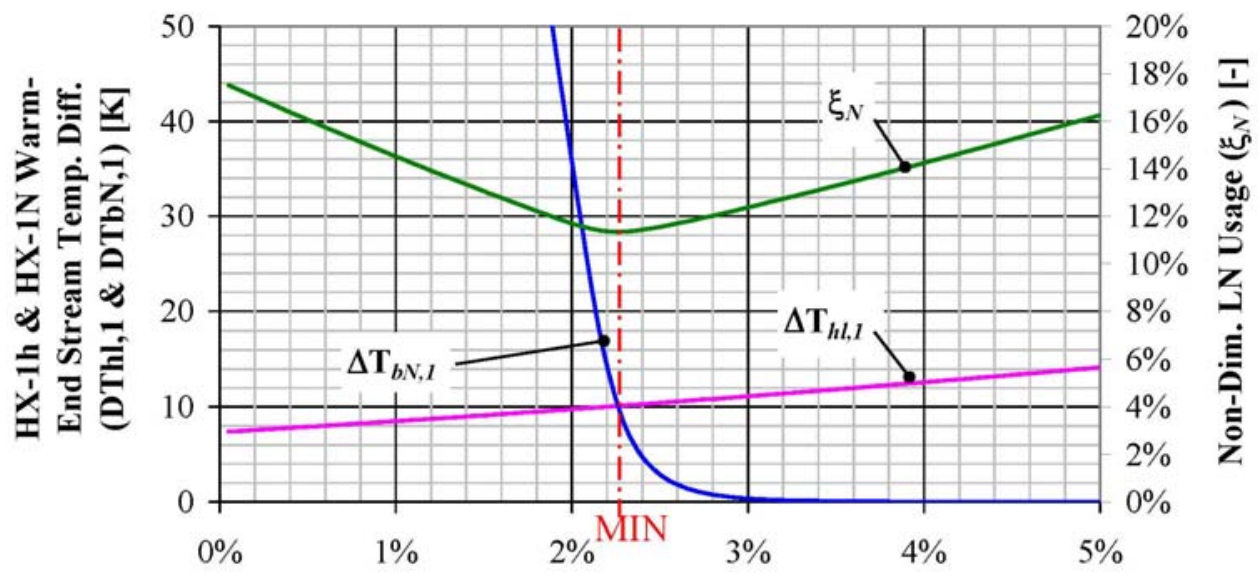

Ratio of Bypass to Total Helium Mass Flow $\left(\xi_{b}\right)[-]$

FIGURE 6. Effect of Helium Bypass Flow Ratio for Fixed HX Size

In practice, the absolute warm end temperatures vary during the day and with the seasons and as such are not a stable and consistent parameter for control. Also, instrumentation errors will have a more significant effect if absolute temperature measurements are used for control. Therefore, it is good practice for stable and consistent control to use temperature differences as the process variable(s). This practice has been validated by successful implementation at JLab since 1994 on helium refrigeration systems of several different manufacturers and in many other systems using LN pre-cooling.

As demonstrated in this paper, it is the size of the helium-helium HX (i.e., HX-1h) that governs the design LN usage. Although, the size of the helium-nitrogen HX (HX-1N) should be reasonable, special attention must be given to the design of the helium-helium HX.

Although the mixed helium temperature just upstream of the helium-nitrogen boiler provides a direct indication of (minimum) LN usage, this signal is typically not available and like all absolute indications is vulnerable to calibration and accuracy errors. So, as presented herein, for a given LN pre-cooler design the helium-helium HX warm-end stream temperature difference is the proper process variable to ensure stability and affect minimal LN usage. Correspondingly, the proper control element is either the helium bypass valve (PCV-B), for Type 2 pre-coolers so equipped, or the LN supply valve (PCV-N) for Type 1 pre-coolers. A cascade control can be added to prevent the helium-nitrogen warm-end stream temperature difference or the $80-\mathrm{K}$ level temperature (for proper operation of the $80-\mathrm{K}$ beds) from becoming excessive.

\section{ACKNOWLEDGEMENTS}

The authors would like to express their appreciation and thanks to the TJNAF management for their support. This work was supported by the U.S. Department of Energy under contract no. DE-AC05-06OR23177.

\section{REFERENCES}

1. Ganni, V., Design of Optimal Helium Refrigeration and Liquefaction Systems, CEC-ICMC 2009 - CSA Short Course Symposium. 